\title{
The Geriatric Nutrition Risk Index versus the Mini- Nutritional Assessment Short Form in predicting postoperative delirium and hospital length of stay among older non-cardiac surgical patients: a prospective cohort study
}

\section{Yanli Zhao}

Sichuan University West China Hospital

Ning Ge ( $\nabla$ grace7733@163.com )

Sichuan University West China Hospital

\section{Dongmei Xie}

Sichuan University West China Hospital

\section{Langli Gao}

Sichuan University West China Hospital

\section{Yanyan Wang}

Sichuan University West China Hospital

Yulin Liao

Guangxi Medical University First Affiliated Hospital

Jirong Yue

Sichuan University West China Hospital

\section{Research article}

Keywords: Geriatric Nutritional Risk Index, Mini-Nutritional Assessment Short Form, Older people, Postoperative delirium, Length of stay, Non-cardiac surgery

Posted Date: February 11th, 2020

DOI: https://doi.org/10.21203/rs.2.17879/v2

License: (c) (1) This work is licensed under a Creative Commons Attribution 4.0 International License. Read Full License

Version of Record: A version of this preprint was published at BMC Geriatrics on March 17th, 2020. See the published version at https://doi.org/10.1186/s12877-020-1501-8. 


\section{Abstract}

Backgrounds: Malnutrition has been shown to be associated with poor prognosis in older surgical patients. Several tools are available for detecting malnutrition. But little is known about their ability to assess risks of postoperative adverse outcomes. The study aimed to compare the ability of the Geriatric Nutritional Risk Index (GNRI) and the Mini-Nutritional Assessment Short Form (MNA-SF) in predicting postoperative delirium (POD) and length of stay (LOS) among older non-cardiac surgical patients. Methods : Prospective study of 288 older non-cardiac surgical patients from the West China Hospital of Sichuan University. Preoperative nutritional status was assessed using the GNRI and MNA-SF, and patients were followed for the occurrence of POD and LOS. Multivariable logistic regression and linear regression analyses were used to identify predictors of these outcomes. The relative performance of the GNRI and MNA-SF as predictors of these outcomes were determined by Receiver Operating Characteristic curves (ROC) analyses and the area under the curve (AUC). Results : Multivariable analysis revealed that preoperative malnutrition by the MNA-SF was significantly associated with POD. Linear regression analysis showed that preoperative low/high nutritional risk of the GNRI and malnutrition by the MNA-SF were independent predictors of prolonged LOS. Moreover, the area under the curve (AUC) of MNA-SF scores for POD was better than GNRI scores (AUC=0.718, 95\%Cl: 0.64-0.80, $P<0.001$ vs AUC $=0.606$, 95\%Cl: 0.52-0.69, $P=0.019$; Delong's test, $P=0.006)$, but the AUC of GNRI scores and MNA-SF scores have no significant difference when predicting prolonged LOS (AUC $=0.611,95 \% \mathrm{Cl}: 0.54-0.69, \mathrm{P}=0.006$ vs AUC $=0.533,95 \% \mathrm{Cl}: 0.45-0.62, \mathrm{P}=0.421$; Delong's test, $\mathrm{P}=0.079$ ). Conclusion : The MNA-SF was more effective than the GNRI at predicting the development of POD, but the two nutrition screening methods have similar performance in predicting prolonged LOS among older non-cardiac surgical patients.

\section{Background}

The rapid aging of the general population is resulting in a greater number of older patients in need of surgery. Malnutrition is a common comorbidity in surgical patients. Advanced age, chronic diseases, reliance upon a large number of drugs, low nutrient intake, reduced appetite, and psychological conditions are risk factors for the development of nutritional deficiencies $(1,2)$. The prevalence of malnutrition in geriatric hospitalized patients has been estimated to range from $30 \%$ to $60 \%$ depending on the population studied and the applied assessment tools $(3,4)$. Despite these high rates of malnutrition, this issue has not received sufficient clinical attention (5). The presence of malnutrition is associated with adverse clinical outcomes, including a higher rate of delirium, prolonged length of stay, morbidity, mortality and increase of healthcare costs (6-8). Furthermore, several studies have found that nutritional intervention can mitigate the risk of delirium and prolonged hospitalization $(9,10)$. Therefore, early nutritional screening in hospitalized patients is important for estimating the risk of nutrition-related complications, especially delirium and length of hospital stay.

There are currently multiple different screening tools available for assessing nutritional status in the older people. Of these tools, the Mini Nutritional Assessment-Short Form (MNA-SF) is recommended by the European Society for Clinical Nutrition and Metabolism (ESPSN), as it has been validated for the 
diagnosis of malnutrition and for prediction of clinical outcomes $(11,12)$. Recently, a novel screening tool, the Geriatric Nutritional Risk Index (GNRI), has been proposed (13). As this screening method is dependent upon objective measurements that do not require patient cooperation, it can be applied in all clinical settings $(14,15)$. The validity of the GNRI for the prediction of short and long-term outcomes has been clearly demonstrated in previous studies $(16,17)$. To date, some studies have compared the ability of different nutritional screening tools to assess malnutrition status, hospital length of stay, mortality, and infection-associated complications in hospitalized patients (17-19). However, studies comparing the ability of the GNRI and MNA-SF in predicting postoperative delirium (POD) and length of hospital stay (LOS) are still scarce.

Thus, the aim of our study was to compare the GNRI with the MNA-SF regarding its ability to predict POD and LOS in older non-cardiac surgical patients.

\section{Methods}

\section{Study design and population}

This prospective cohort study was conducted in the West China Hospital of Sichuan University from April to June of 2015. Eligible patients were 70 years or older, scheduled for elective non-cardiac surgery, and had an anticipated length of stay of at least 3 days. Exclusion criteria included: (1) severe hearing impairment, (2) inability to communicate because of severe dementia or psychiatric illness, (3) a terminal condition with life expectancy of less than 6 months (eg, metastatic cancer, pancreatic cancer, or receiving end-of-life care), (4) the presence of delirium at baseline.

The study was approved by the Institutional Review Boards of West China Hospital, Sichuan University, and was carried out according to the principles of the Declaration of Helsinki. All the participants provided written informed consent.

\section{Data collection and Sample size}

All patients were preoperatively assessed by trained research nurses within 48 hours of admission, and the following data were collected: age, gender, and type of surgery (orthopedic, general, thoracic). Delirium was initially screened using the Confusion Assessment Method (CAM) on admission . Comorbidities were evaluated on admission using the $\mathrm{CCl}$ (Charlson Comorbidity Index) (20). Functional status was evaluated using the Barthel Index (21). Preoperative pain was measured using the Facial Scale (range $0-10$ ) via patient interview (22). The presence of depressive symptoms was assessed using the 15-item version of the validated Geriatric Depression Scale (GDS-15) (23).

Previous results suggested that the incidence of POD in older patients was $13 \%-50 \%$ (24). We hypothesized that the incidence rate in our study was $20 \%$ and the AUC was not less than 0.7 . Assuming a type I error of $5 \%$, a type II error of $10 \%$, and taking design into account, we estimated the sample size to be 135 . Allowing for $20 \%$ attrition, we increased the sample size to 168 . 


\section{Nutritional assessment}

The GNRI and MNA-SF were used to assess preoperative nutritional status. The GNRI, which was adapted from the Nutritional Risk Index (NRI) designed by Buzby et al. (25), is a simple nutritionl screening tool to evaluate nutritional-related complications. The index was calculated as follows (13): GNRI $=1.489$ serum albumin $(\mathrm{g} / \mathrm{L})+41.7$ present weight/ideal weight $(\mathrm{kg})$. Ideal body weight was derived according to the following equations of Lorentz (13): ideal weight for men $=0.75$ height $(\mathrm{cm})-62.5$, ideal weight for women $=0.60$ height $(\mathrm{cm})-40$. Unlike the original categorization in four classes proposed by Bouillanne et al. (13). The participants in our study were stratified into three categories similar to previous study: no risk (GNRI > 98), low risk (92-98), severe/moderate risk (GNRI < 92) (17). We merged the category of severe risk $(\mathrm{GNRI}<82)$ and moderate risk (GNRI 82 to $<92)$ into one single category, as these two categories were associated with a similar increased odds of complications (17). The MNA-SF is a validated, sensitive, reliable screening tool which consists of six domains: appetite or eating problems in the past 3 months, weight loss in the past 3 months, mobility impairment, acute illness/stress, dementia or depression, and BMI. Total scores of MNA-SF range from 0 to 14, and patients were divided into the following three categories according to the following cut-offs: well-nourished (12-14), risk of malnutrition (8-11), malnourished (0-7) (26).

\section{Outcomes}

All patients were followed for the occurrence of POD and LOS. The CAM was used daily by the trained research assessors for delirium within 7 days after surgery. The CAM is based on the following four features (27): (i) acute onset and fluctuating course; (ii) inattention; (iii) disorganized thinking; (iv) altered level of consciousness. A positive diagnosis of delirium required the presence of both items (i) and (ii), and either item (iii) or (iv). LOS was defined as the number of days in the hospital from the day of admission to the day of discharge. Prolonged LOS was defined as LOS beyond the 75th percentile of its distribution (computed to $\geq 22$ days in our study) (28).

\section{Statistical analysis}

Continuous variables were expressed as means with the standard deviation (SD) for normally distributed data, and as medians with the interquartile range (IQR) for non-normally distributed data. ANOVAs and Kruskall-Wallis tests were used for between-group comparisons of continuous variables with normal and non-normal distributions, respectively. Categorical variables were expressed as the number of cases and percentages, and were compared using Chi-squared tests.

A multivariable logistic regression model was performed to investigate the association between the two screening methods and POD, and a linear regression model was used for LOS. The logistic regression model was adjusted for age, sex, preoperative pain, depression, Barthel Index and CCl. The linear regression model was adjusted for age, sex, Barthel Index and preoperative pain. The GNRI and MNA-SF were included in regression models as continuous and categorical variables respectively. To fulfill the purpose of the study, the discriminative ability of each nutritional screening tool for the outcomes was 
assessed by the Receiver Operating Characteristic (ROC) curves and the area under the curve (AUC). Comparisons between AUCs were performed by Delong's test (29). Furthermore, the sensitivity, specificity and likelihood ratios (positive and negative) of different GNRI and MNA-SF cut-offs were calculated.

Statistical analyses were performed using IBM SPSS version 21.0 and MedCalc version 19.1. A $P$ value < 0.05 was considered statistically significant.

\section{Results}

\section{Baseline characteristic of patients}

A total of 348 patients were admitted to our hospital. 28 and 32 patients were excluded due to cancelling scheduled surgery and incomplete data, respectively. Finally, 288 subjects were included in the analyses. The median age of these patients was 74 years (IQR 72-28), and 148 patients (51.4\%) were male. Of the overall population, 49 (17\%) developed POD, and median LOS was 14 days (IQR 10-21). In our study, the number of patients who underwent general, orthopedic and thoracic surgery were $189(65.6 \%), 71$ (24.7\%) and $28(9.7 \%)$, respectively.

\section{The characteristics of the population as determined by the MNA-SF and GNRI}

The characteristics of the patients screened by the MNA-SF and GNRI are shown in tables 1 and 2. According to the GNRI, $29.5 \%$ and $15.6 \%$ of patients were low risk and high risk, respectively. Based on the MNA-SF, $34 \%$ and $14.2 \%$ of patients were at risk of malnutrition and malnourished, respectively. There were significant differences in the Barthel Index, POD incidence, and LOS among different GNRI and MNASF categories. By a post-hoc comparison, we found that albumin levels was significantly lower in subjects who were malnourished and at risk of malnutrition compared to those who were well-nourished according to the MNA-SF.

Table 1 Characteristics of the studied population according to the Geriatric Nutritional Risk Index (GNRI)

\begin{tabular}{|c|c|c|c|c|c|}
\hline Characteristic & Total & $\begin{array}{l}\text { High Risk } 92 \\
(\mathrm{n}=45)\end{array}$ & $\begin{array}{l}\text { Low Risk 92-98 } \\
\qquad(\mathrm{n}=85)\end{array}$ & $\begin{array}{c}\text { No Risk > 98 } \\
\quad(n=158)\end{array}$ & $P$-value ${ }^{\text {a }}$ \\
\hline Age (years), median (IQR) & $74(72-78)$ & $75(73-79)$ & $75(72-78)$ & $74(71-77)$ & 0.086 \\
\hline Male gender, n (\%) & $148(51.4)$ & $24(53.3)$ & $36(42.4)$ & $88 \square 55.7 \square$ & 0.134 \\
\hline Preoperative pain, n (\%) & $169(58.7)$ & $26(57.8)$ & $56(65.9)$ & $87(55.1)$ & 0.261 \\
\hline Depression, n (\%) & $19(6.6)$ & $4(8.9)$ & $5(5.9)$ & $10(6.3)$ & 0.79 \\
\hline \multicolumn{6}{|l|}{ CCI, n (\%) } \\
\hline Mild $(\leq 2)$ & $214(74.3)$ & $34(75.6)$ & $58(68.2)$ & $122(77.2)$ & \\
\hline Moderate (3-4) & $52(18.1)$ & $7(15.6)$ & $21(24.7)$ & $24(15.2)$ & 0.546 \\
\hline Severe $(\geq 5)$ & $22(7.6)$ & $4(8.9)$ & $6(7.1)$ & $12(7.6)$ & \\
\hline GNRI score, mean \pm SD & $98.98 \pm 8.46$ & $85.09 \pm 5.45^{b}$ & $95.12 \pm 2.17^{b}$ & $105.01 \pm 4.49^{b}$ & $\square 0.001$ \\
\hline MNA-SF score, med (IQR) & $12(9-13)$ & $9(7-11)$ & $10(8-12)$ & $12(11-14)$ & $\square 0.001$ \\
\hline Barthel Index, median (IQR) & $100(90-100)$ & $95(72-100)$ & $100(87-100)$ & $100(95-100)$ & 0.010 \\
\hline Postoperative delirium, n (\%) & $49(17.0)$ & $14(31.1)$ & $11(12.9)$ & $24(15.2)$ & 0.021 \\
\hline Length of stay, med (IQR) & $14(21-10)$ & $17(12-21)$ & $17(12-23)$ & $13(9-18)$ & $\square 0.001$ \\
\hline
\end{tabular}


BMI body mass index, CCI Charlson Comorbidity Index, MNA-SF Mini Nutritional Assessment-Short Form.

Notes: ${ }^{\mathrm{a}} p$ values according to ANOVA, Kruskall-Wallis or Chi-square tests;

$\mathrm{b}$ Significantly different from the other groups by post-hoc comparison.

Table 2 Characteristics of the studied population according to the Mini-Nutritional Assessment Short Form (MNA-SF)

\begin{tabular}{lcccr}
\hline Characteristic & $\begin{array}{c}\text { Malnourished 7 } \\
(\mathrm{n}=41)\end{array}$ & $\begin{array}{c}\text { At risk 8-11 } \\
(\mathrm{n}=98)\end{array}$ & $\begin{array}{c}\text { Well nourished 12-14 } \\
(\mathrm{n}=149)\end{array}$ & $P_{\text {-value }}{ }^{\text {a }}$ \\
\hline Age (years), median (IQR) & $76(73-78)$ & $75.5(72-78)$ & $73(71-77)$ & 0.078 \\
Male gender, n (\%) & $22(53.7)$ & $49(50.0)$ & $77(51.7 \square$ & 0.921 \\
Preoperative pain, n (\%) & $30(24.1)$ & $59(60.2)$ & $80(53.7)$ & 0.075 \\
BMI $\left(\mathrm{kg} / \mathrm{m}^{2}\right)$, mean $\pm \mathrm{SD}$ & $19.45 \pm 2.55^{\mathrm{b}}$ & $21.42 \pm 3.07^{\mathrm{b}}$ & $23.70 \pm 2.88^{\mathrm{b}}$ & $\square 0.001$ \\
Albumin (g/L), mean $\pm \mathrm{SD}$ & $36.93 \pm 5.13^{\mathrm{b}}$ & $39.79 \pm 4.73^{\mathrm{b}}$ & $41.21 \pm 4.48^{\mathrm{b}}$ & $\square 0.001$ \\
MNA-SF score, med (IQR) & $6(5-7)^{\mathrm{b}}$ & $10(9-11)^{\mathrm{b}}$ & $13(12-14)^{\mathrm{b}}$ & $\square 0.001$ \\
Barthel Index, median (IQR) & $95(77-100)$ & $100(88-100)$ & $100(95-100)$ & 0.010 \\
Postoperative delirium, n (\%) & $16(39.0)$ & $20(20.4)$ & $13(8.7)$ & 0.004 \\
Length of stay, med (IQR) & $19(14-23)$ & $14(10-19)$ & $14(10-20)$ & 0.022 \\
\hline
\end{tabular}

BMI body mass index, CCI Charlson Comorbidity Index, GNRI Geriatric Nutritional Risk Index.

Notes: ${ }^{\mathrm{a}} p$ values according to ANOVA, Kruskall-Wallis or Chi-square tests;

$\mathrm{b}$ Significantly different from the other groups by post-hoc comparison.

\section{Multivariable logistic regression and linear regression analysis}

In the multivariable model, the malnourished category of the MNA-SF was independent risk factor for POD after adjustment of age, sex, preoperative pain, depression, Barthel Index and CCl, while the GNRI was not predictor for POD (Table 3). In the linear regression, prolonged LOS was significantly associated with low and high risk of the GNRI, but only with malnourished category by the MNA-SF after adjustment of age, sex, Barthel Index and preoperative pain (Table 3). When modeled as a continuous variable, MNASF was independent predictor of prolonged LOS and POD, while the GNRI (modeled as a continuous variable) was only significantly correlated with LOS.

Table 3 Multivariable logistic regression and linear regression analyses for the occurrence of postoperative delirium and length of stay

\begin{tabular}{|c|c|c|c|c|}
\hline & \multicolumn{2}{|c|}{ Postoperative delirium } & \multicolumn{2}{|l|}{ Length of stay } \\
\hline & Adjusted OR $(95 \% \text { CI })^{\mathrm{a}}$ & $P$-value & $\beta(95 \% \mathrm{CI})^{\mathrm{b}}$ & $P$-value \\
\hline \multicolumn{5}{|l|}{ GNRI categories ${ }^{\mathrm{C}}$} \\
\hline No risk ( $\square 98)$ & Reference & & Reference & \\
\hline Low risk (92-98) & $0.61(0.26-1.43)$ & 0.255 & $4.91(2.43-7.39)$ & $<0.001$ \\
\hline High risk (ロ92) & $2.22(0.92-5.37)$ & 0.077 & $4.10(0.95-7.25)$ & 0.011 \\
\hline GNRI scores ${ }^{d}$ & $0.96(0.92-1.00)$ & 0.077 & $-0.24(-0.37$ to -0.11$)$ & $<0.001$ \\
\hline \multicolumn{5}{|l|}{ MNA-SF categories ${ }^{C}$} \\
\hline Well nourished (12-14) & Reference & & Reference & \\
\hline At risk $(8-11)$ & $1.95(0.86-4.39)$ & 0.109 & $0.87(-1.55$ to 3.29$)$ & 0.48 \\
\hline Malnourished $(\leq 7)$ & $4.06(1.62-10.18)$ & 0.003 & $4.23(0.91-7.54)$ & 0.013 \\
\hline MNA-SF scores $d$ & $0.82(0.72-0.92)$ & 0.001 & $-0.52(-0.93$ to -0.11$)$ & 0.014 \\
\hline
\end{tabular}


OR odds ratio, CI confidence interval, GNRI Geriatric Nutritional Risk Index, MNA-SF Mini-Nutritional Assessment Short Form, CCI Charlson Comorbidity Index.

Notes: a Adjusted for age, sex, CCI, depression, Barthel Index and preoperative pain in multivariable logistic regression model;

b Adjusted for age, sex, Barthel Index and preoperative pain in linear regression model;

C Modeled as categories variables;

d Modeled as continuous variables.

\section{ROC curve analysis}

Based on the ROC curve analyses and Delong's test, MNA-SF scores showed higher AUC in predicting POD than GNRI scores (Table 4, Fig. 1). In addition, the AUC of MNA-SF scores was significantly higher than GNRI scores (Delong's test, $\mathrm{P}=0.006$ ). Although the AUC of GNRI scores for prolonged LOS was better than MNA-SF scores (Table 4, Fig. 2), there was no significant difference in determining prolonged LOS (Delong's test, $\mathrm{P}=0.079$ ). As shown in Table 4, both $\mathrm{MNA}-\mathrm{SF}<8$ and $\mathrm{GNRK}<92$ exhibited satisfactory specificity values $({ } 60 \%)$ in predicting POD and prolonged LOS. However, the sensitivity values of the two categories were below adequate $(<80 \%)$.

Table 4 Sensitivity, specificity, positive likelihood ratios (PLR), negative likelihood ratios (NLR) and area under the curve (AUC) of nutritional screening tools

\begin{tabular}{lllllll}
\hline & Sensitivity & Specificity & PLR & NLR & AUC (95\% CI) & $P($ AUC) \\
\hline $\begin{array}{l}\text { Postoperative delirium } \\
\text { GNRI }\end{array}$ & & & & & & \\
$\quad$ Scores $\square 92$ & $28.6 \%$ & $87.0 \%$ & 2.20 & 0.82 & $0.606(0.52-0.69)$ & 0.019 \\
$\quad$ Scores $\leq 98$ & $51.0 \%$ & $56.1 \%$ & 1.16 & 0.87 & & \\
MNA-SF & & & & & & \\
$\quad$ Scores $\square 8$ & $32.7 \%$ & $89.5 \%$ & 3.11 & 0.75 & $0.718(0.64-0.80)$ & $\square 0.001$ \\
$\quad$ Scores $\square 12$ & $73.5 \%$ & $56.9 \%$ & 1.71 & 0.47 & & \\
$\quad$ Prolonged length of stay & & & & & & \\
GNRI & & & & & & \\
$\quad$ Scores $\square 92$ & $16.9 \%$ & $84.8 \%$ & 1.11 & 0.98 & $0.611(0.54-0.69)$ & 0.006 \\
$\quad$ Scores $\leq 98$ & $61.5 \%$ & $59.6 \%$ & 1.52 & 0.65 & & \\
$\quad$ MNA-SF & & & & & & \\
$\quad$ Scores $\square 8$ & $23.1 \%$ & $88.3 \%$ & 1.97 & 0.87 & $0.533(0.45-0.62)$ & 0.421 \\
$\quad$ Scores $\square 12$ & $50.8 \%$ & $52.5 \%$ & 1.07 & 0.94 & & \\
\hline
\end{tabular}

CI confidence interval, GNRI Geriatric Nutritional Risk Index, MNA-SF Mini-Nutritional Assessment Short Form.

\section{Discussion}

Early and accurate identification of patients at risk of malnutrition-associated complications could effectively guide surgical practices. Although there are number of malnutrition assessment tools available designed to determine the risk of postoperative adverse outcomes, there are at present few studies evaluating the validity of the MNA-SF and the GNRI as predictors of nutrition-related morbidity in a surgical setting. To the best of our knowledge, this is the first prospective study to compare the ability of the GNRI and MNA-SF in predicting POD and LOS among older patients undergoing non-cardiac surgery. Our results revealed that the MNA-SF seemed to be better at predicting POD, whereas the MNA-SF and the GNRI have similar ability in predicting the risk of POD among older non-cardiac surgical patients. 
In this study, the prevalence of high risk of malnutrition determined by the GNRI was lower than that reported by Duran et al. (30), in which $32.5 \%$ of 40 elderly patients in acute geriatric ward were at high nutritional risk. Patients in the acute geriatric ward were more likely to be have severe and acute illness than surgical patients, which may explain these differences. The MNA-SF detected malnutrition in $14.2 \%$ of patients in the current study. Another study found a similar prevalence of malnutrition by the MNA-SF (31).

Serum albumin is generally considered as a crude indicator for nutritional status, particularly among patients with chronic conditions; a low level of serum albumin could indicate either poor nutritional status or inflammatory status or both (32). Previous studies have demonstrated that the MNA-SF and the GNRI were more suitable for assessing nutritional status than serum albumin $(33,13)$. In addition, the GNRI and MNA-SF had better performance in predicting adverse outcomes than serum albumin $(34,35)$. Thus, more comprehensive and systematic nutritional screening methods, such as the GNRI and the MNA-SF, are needed to evaluate nutritional status or nutrition-related complications among surgical patients.

According to the present results, the MNA-SF outperformed GNRI in predicting POD among elderly noncardiac surgical patients. To date, only one study by Sugita, et al. compared different screening tools, including the GNRI, PNI and CONUT, for prediction of delirium in coronary intensive care unit patients and they observed no a significant association between GNRI and delirium (36), which is similar to our study. Recently, Chu et al. and Mazzola et al. used the MNA-SF as a screening tool among orthopedic surgical patients and detected a significant association between MNA-SF and POD $(31,37)$. In addition, previous studies have found that the nutritional intervention could reduce the incidence of postoperative delirium and shorten its duration in older surgical patients $(10,38,39)$. Our results were consistent with these, and confirmed the value of the MNA-SF as a predictor of POD.

The superiority of the MNA-SF as a predictor of POD may be explained by the fact that it incorporates neuropsychological, functional and psychological parameters, all of which are risk factors for the development of delirium. Although depression and dementia, included in the items of the MNA-SF, are also part of the $\mathrm{CCl}$ and GDS-15. We have excluded patients with the severe dementia on admission and the number of patients with severe depression is low in our study. Moreover, unlike the dementia and mild/moderate depression items of the MNA-SF, severe dementia and depression should be diagnosed by the specialist.

The GNRI and the MNA-SF have similar performance in predicting prolonged LOS in the present study. This is different from findings reported in Abd-EL-Gawad et al., in which the GNRI was found more effective than the MNA in the evaluation of prolonged hospitalization(19). Recently, many studies have used nutritional screening tools to predict hospitalization period (40-42). There is accumulating evidence that early nutritional screening in older patients who might benefit from nutritional treatment may result in a shorter $\operatorname{LOS}(6,8,9)$. These studies support that malnutrition is useful for predicting LOS. However, the causal relation between nutritional status and LOS remains unclear; rather, length of hospital stay may reflect the severity of underlying disease. 
Indeed, the MNA-SF and GNRI are relatively simple screening tools that can be rapidly applied to clinical practice. Advantages of the MNA-SF are its high sensitivity in regard to nutritional assessment and the lack of requirement for biochemical tests. However, it cannot be used in patients receiving parenteral nutrition or who have poor cognitive function (3). The GNRI was designed to overcome the subjective bias of the MNA and the difficulties in acquiring usual weight and standing height $(13,15)$. It may be useful in surgical patients with cognitive impairment because it is an objective index based only on weight, height, and serum albumin levels (13). Further studies comparing the GNRI and the MNA-SF as predictors of adverse outcomes are required to validate their utility.

This study has several limitations. First, this is a single-center study with a small sample size, the results may not represent a general population of older surgical patients. Multicenter and larger studies are required in the future. Second, the 7-day duration of screening for delirium was chosen to balance the peak days of delirium onset in the population (POD occurred 1-3 days after surgery) against the practical constraints of our resources. Third, due to realistic constraints of our resources, intraoperative data and postoperative data in Intensive Care Units were not included in our study. Fourth, Patients with risk of malnutrition by the MNA-SF were not screened using the MNA owing to practical constraints. The prevalence of malnutrition may be underestimated. Fifth, we did not collect information regarding the occurrence of in-hospital mortality and assessment of functional status at discharge.

\section{Conclusion}

The current study demonstrated that the MAN-SF is more reliable as a means of evaluating patients for the development of POD, whereas the MNA-SF and the GNRI have similar performance in predicting prolonged LOS. Present results may help clinicians to choose appropriate nutrition screening method to predict different outcomes. It also highlights the importance of early detection and timely intervention for patients who are at risk of undernutrition, in order to prevent negative postoperative outcomes. In the future, more studies comparing the ability of different nutritional screening tools in predicting adverse outcomes among surgical patients are needed.

\section{Abbreviations}

GNRI, Geriatric Nutritional Risk Index; MNA-SF, Mini Nutritional Assessment Short Form; POD, postoperative delirium; LOS, length of stay; $\mathrm{CCl}$, Charlson Comorbidity Index; CAM, Confusion Assessment Method.

\section{Declarations}

\section{Acknowledgments}

We would like to thank the staff of the Department of Geriatrics Medicine, West China Hospital and all participants for their great contribution. 


\section{Author Contributions}

JR Yue and YL Zhao conceptualized and designed the study; YL Liao and YY Wang. coordinated data collection; YL Zhao, DM Xie, LL Gao and NG. analyzed data, prepared and reviewed figures. YL Zhao and JR Yue wrote the original draft; All authors reviewed the manuscript.

\section{Funding}

This study was supported by Health Research of Cadres in Sichuan province (grant number: SCR2019102, 2019-109, 2017-111, 2019-106, ZH2019-102) and Sichuan Science and Technology Program (grant number: 2015SZ0091, 2018SZ0252, 2019YFS0448, 2019YJ0033).

\section{Availability of data and materials}

The datasets used for the current study are available from the corresponding author upon reasonable request.

\section{Ethics approval and consent to participate}

Ethics approval was obtained form the Institutional Review Boards of West China Hospital, Sichuan University. All the participants provided written informed consent.

\section{Consent for publication}

Not applicable.

\section{Competing interests}

The authors declare that they have no competing interests.

\section{Author details}

${ }^{1}$ Department of Geriatrics and National Clinical Research Center for Geriatrics, West China Hospital of Sichuan University, Chengdu, Sichuan, China. ${ }^{2}$ Department of Respiratory Diseases, First Affiliated Hospital of Guangxi Medical University, Nanning, Guangxi, China.

\section{References}

1. Drewnowski A, Shultz JM. Impact of aging on eating behaviors, food choices, nutrition, and health status. J Nutr Health Aging. 2001;5(2):75-9.

2. Guidelines for the use of parenteral and enteral nutrition in adult and pediatric patients. JPEN J Parenter Enteral Nutr. 2002;26:1sa-138sa.

3. Poulia KA, Yannakoulia M, Karageorgou D, Gamaletsou M, Panagiotakos DB, Sipsas NV, Zampelas A. Evaluation of the efficacy of six nutritional screening tools to predict malnutrition in the elderly. Clin 
Nutr. 2012;31(3):378-85.

4. Gazzotti C, Arnaud-Battandier F, Parello M, Farine S, Seider L, Albert A, Petermans J. Prevention of malnutrition in older people during and after hospitalisation: results from a randomised controlled clinical trial. Age Ageing. 2003;32(3): 321-5.

5. Barker LA, Gout BS, Crowe TC. Hospital malnutrition: prevalence, identification and impact on patients and the healthcare system. Int J Environ Res Public Health. 2011;8(2):514-27.

6. Correia MI, Waitzberg DL. The impact of malnutrition on morbidity, mortality, length of hospital stay and costs evaluated through a multivariate model analysis. Clin Nutr. 2003;22(3):235-9.

7. Ringaitiene D, Gineityte D, Vicka V, Zvirblis T, Sipylaite J, Irnius A, Ivaskevicius J, Kacergius T. Impact of malnutrition on postoperative delirium development after on pump coronary artery bypass grafting. J Cardiothorac Surg. 2015;10:74.

8. Allard JP, Keller H, Jeejeebhoy KN, Laporte M, Duerksen DR, Gramlich L, Payette H, Bernier P, et al. Decline in nutritional status is associated with prolonged length of stay in hospitalized patients admitted for 7 days or more: A prospective cohort study. Clin Nutr. 2016;35(1):144-52.

9. Kyle UG, Genton L, Pichard C. Hospital length of stay and nutritional status. Curr Opin Clin Nutr Metab Care. 2005;8(4):397-402.

10. Olofsson B, Stenvall M, Lundstrom M, Svensson O, Gustafson Y. Malnutrition in hip fracture patients: an intervention study. J Clin Nurs. 2007;16(11):2027-38.

11. Kondrup J, Allison SP, Elia M, Vellas B, Plauth M. ESPEN guidelines for nutrition screening 2002. Clin Nutr. 2003;22(4):415-21.

12. Guigoz Y, Lauque S, Vellas BJ. Identifying the elderly at risk for malnutrition. The Mini Nutritional Assessment. Clin Geriatr Med. 2002;18(4):737-57.

13. Bouillanne O, Morineau G, Dupont C, Coulombel L, Vincent JP, Nicolis I, Benazeth S, Cynober L, Aussel C. Geriatric Nutritional Risk Index: a new index for evaluating at-risk elderly medical patients. Am J Clin Nutr. 2005;82(4):777-83.

14. Cereda E, Pedrolli C. The Geriatric Nutritional Risk Index. Curr Opin Clin Nutr Metab Care. 2009;12(1):1-7.

15. Cereda E, Limonta D, Pusani C, Vanotti A. Feasible use of estimated height for predicting outcome by the geriatric nutritional risk index in long-term care resident elderly. Gerontology. 2007;53(4):184-6.

16. Cereda E, Limonta D, Pusani C, Vanotti A. Geriatric nutritional risk index: a possible indicator of shortterm mortality in acutely hospitalized older people. J Am Geriatr Soc. 2006;54(6):1011-2.

17. Cereda E, Pusani C, Limonta D, Vanotti A. The ability of the Geriatric Nutritional Risk Index to assess the nutritional status and predict the outcome of home-care resident elderly: a comparison with the Mini Nutritional Assessment. Br J Nutr. 2009;102(4):563-70.

18. Raslan M, Gonzalez MC, Dias MC, Nascimento M, Castro M, Margues P, Segatto S, Torrinhas RS, et al. Comparison of nutritional risk screening tools for predicting clinical outcomes in hospitalized patients. Nutrition. 2010;26(7-8):721-6. 
19. Abd-El-Gawad WM, Abou-Hashem RM, El Maraghy MO, Amin GE. The validity of Geriatric Nutrition Risk Index: simple tool for prediction of nutritional-related complication of hospitalized elderly patients. Comparison with Mini Nutritional Assessment. Clin Nutr. 2014;33(6):1108-16.

20. Charlson ME, Pompei P, Ales KL, MacKenzie CR. A new method of classifying prognostic comorbidity in longitudinal studies: development and validation. J Chronic Dis. 1987;40(5):373-83.

21. Mahoney Fl, Barthel DW. Functional evaluation: The Barthel Index. Md State Med J. 1965;14:61-5.

22. Buchanan $\mathrm{H}$, Niven N. Validation of a Facial Image Scale to assess child dental anxiety. Int $\mathrm{J}$ Paediatr Dent. 2002;12(1):47-52.

23. Nyunt MS, Fones C, Niti M, Ng TP. Criterion-based validity and reliability of the Geriatric Depression Screening Scale (GDS-15) in a large validation sample of community-living Asian older adults. Aging Ment Health. 2009;13(3):376-82.

24. Inouye SK, Westendorp RG, Saczynski JS. Delrium in elderly people. Lancet. 2014;383(9920):911922.

25. Buzby GP, Knox LS, Crosby LO, Eisenberg JM, Haakenson CM, McNeal GE, Page CP, Peterson OL, et al. Study protocol: a randomized clinical trial of total parenteral nutrition in malnourished surgical patients. Am J Clin Nutr. 1998;47(2 Suppl):366-81.

26. Cohendy R, Rubenstein LZ, Eledjam JJ. The Mini Nutritional Assessment-Short Form for preoperative nutritional evaluation of elderly patients. Aging (Milano). 2001;13(4):293-7.

27. Inouye SK, van Dyck CH, Alessi CA, Balkin S, Siegal AP, Horwiz RI. Clarifying confusion: the confusion assessment method. A new method for detection of delirium. Ann Intern Med. 1990;113(12):941-8.

28. Caccialanza R, Klersy C, Cereda E, Cameletti B, Bonoldi A, Bonardi C, Marinelli M, Dionigi P. Nutritional parameters associated with prolonged hospital stay among ambulatory adult patients. CMAJ. 2010;182(17):1843-9.

29. DeLong ER, DeLong DM,Clarke-Pearson DL. Compring the area under two or more correlated receiver operating curves: a nonparametric approach. Biometrics. 1988;44(3):837-45.

30. Durán Alert P, Milà Villarroel R, Formiga F, Virgili Casas N, Vilarasau Farré C. Assessing risk screening methods of malnutrition in geriatric patients: Mini Nutritional Assessment (MNA) versus Geriatric Nutritional Risk Index (GNRI). Nutr Hosp. 2012;27(2):590-598.

31. Chu CS, Liang CK, Chou MY, Lin YT, Hsu CJ, Chou PH, Chu CL. Short-Form Mini Nutritional Assessment as a useful method of predicting the development of postoperative delirium in elderly patients undergoing orthopedic surgery. Gen Hosp Psychiatry. 2016;38:15-20.

32. Roubenoff R. Sarcopenia and its implications for the elderly. Eur J Clin Nutr. 2000;54 Suppl 3:S40S47.

33. Zhou J, Wang M, Wang H, Chi Q. Comparison of two nutrition assessment tools in surgical elderly inpatients in Northern China. Nutr J. 2015;14:68.

34. Nishi I, Seo Y, Hamada-Harimura Y, et al. Geriatric nutritional risk index predicts all-cause deaths in heart failure with preserved ejection fraction. ESC Heart Fail. 2019;6(2):396-405. 
35. Helminen H, Luukkaala T, Saarnio J, Nuotio M. Comparison of the Mini-Nutritional Assessment short and long form and serum albumin as prognostic indicators of hip fracture outcomes. Injury. 2017;48(4):903-908.

36. Sugita Y, Miyazaki T, Shimada K, Shimizu M, Kunimoto M, Ouchi S, Aikawa T, Kadoguchi M, et al. Correlation of Nutritional Indices on Admission to the Coronary Intensive Care Unit with the Development of Delirium. Nutrients. 2018;10(11).

37. Mazzola P, Ward L, Zazzetta S, Broggini V, Anzuini A, Valcarcel B, Brathwaite JS, Pasinetti GM,et al. Association Between Preoperative Malnutrition and Postoperative Delirium After Hip Fracture Surgery in Older Adults. J Am Geriatr Soc. 2017;65(6):1222-8.

38. Harari D, Hopper A, Dhesi J, Babic-Illman G, Lockwood L, Martin F. Proactive care of older people undergoing surgery ('POPS'): designing, embedding, evaluating and funding a comprehensive geriatric assessment service for older elective surgical patients. Age Ageing. 2007;36(2):190-6.

39. Lundstrom M, Olofsson B, Stenvall M, Karlsson S, Nyberg L, Englund U, Borssen B, Svensson O, Gustafson Y. Postoperative delirium in old patients with femoral neck fracture: a randomized intervention study. Aging Clin Exp Res. 2007;19(3):178-86.

40. Kyle UG, Genton L, Pichard C. Hospital length of stay and nutritonal status. Curr Opin Clin Nutr Metab Care. 2005;8(4):397-402.

41. Ruxton CHS, Gordon J, Kirkwood L, McMillan B, Ryan E. Pichard C. Risk of malnutrition in a sample of acute and long stay NHS Fife in-patients: an audit. J Hum Nutr Diet. 2008;21(1):81-90.

42. Gartner S, Kraft M, Kruger J, Vogt LJ, Fiene M, Mayerle J, Aghdassi AA, Steveling A, et al. Geriatric nutritional risk index correlates with length of hospital stay and inflammatory markers in older inpatients. Clin Nutr. 2017;36(4):1048-1053.

\section{Figures}




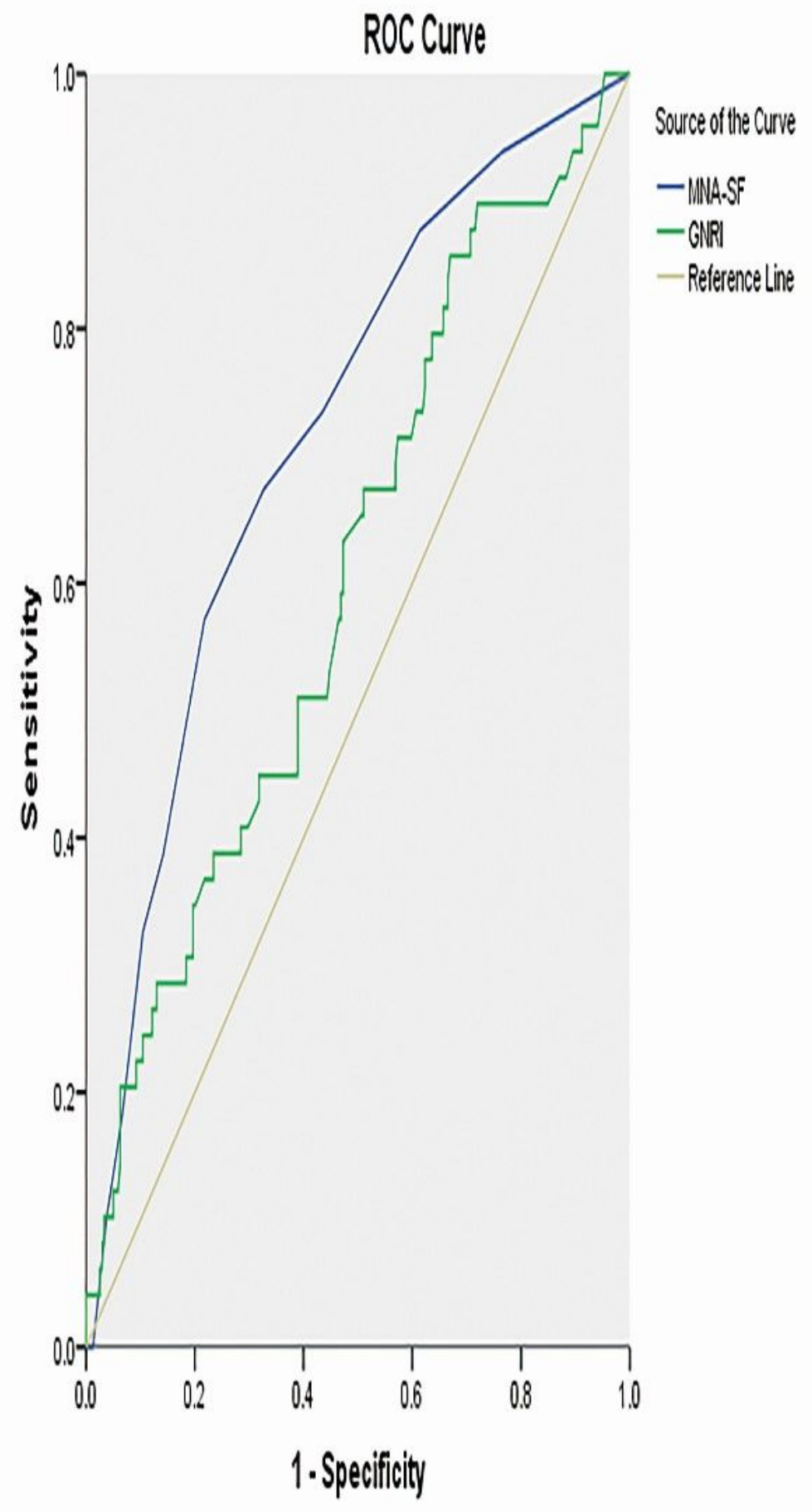

Diagonal segmentits are produced by tiess.

\section{Figure 1}

Receiver operator curve (ROC) of the GNRI and MNA-SF scores for 461 postoperative delirium 


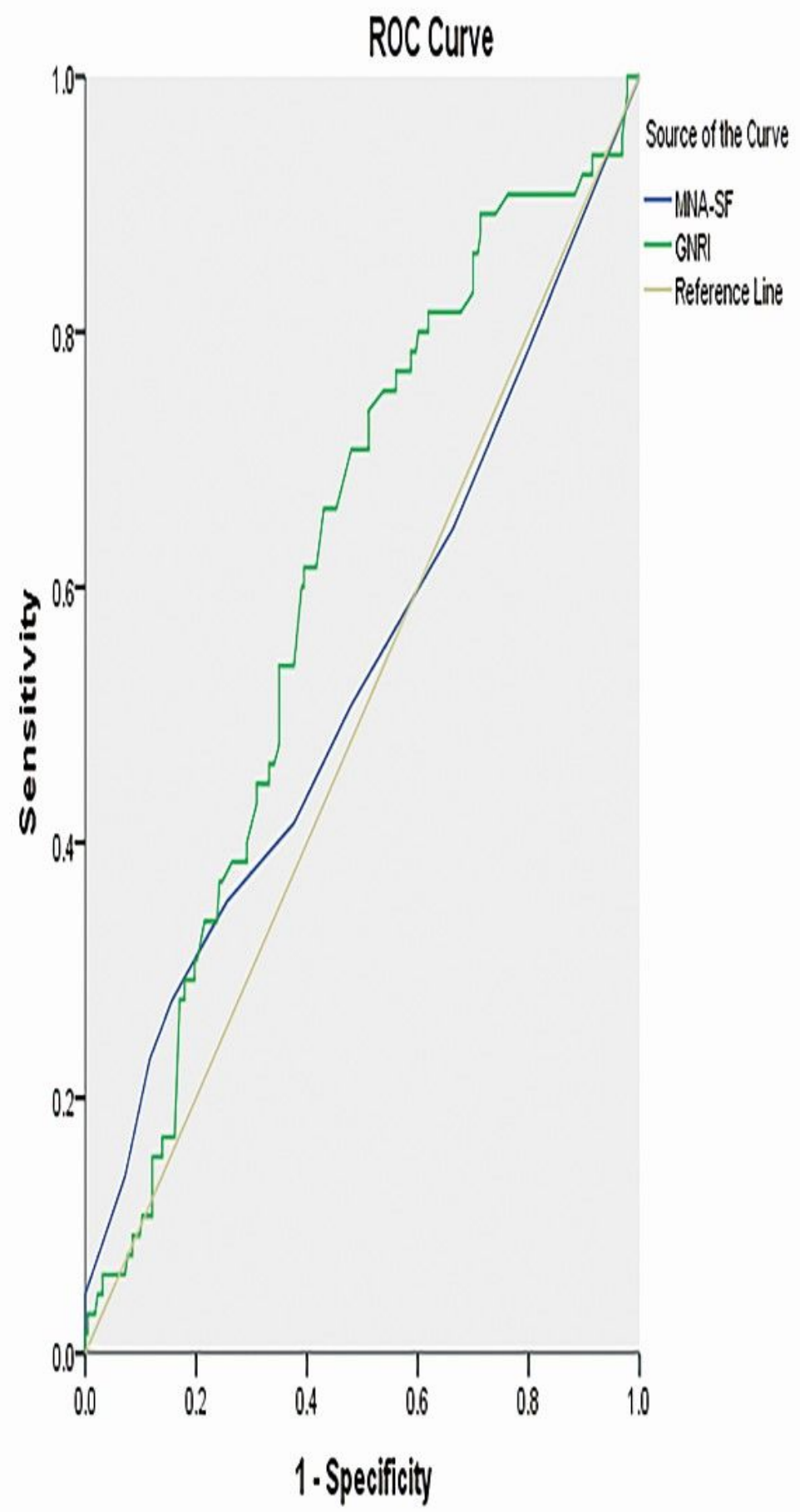

Diagonal segmentis are produced by ties.

Figure 2

Receiver operator curve (ROC) of the GNRI and MNA-SF scores for 463 prolonged length of stay 